\title{
Teoría marxista y transformación de valores en precios, 117 años de controversia
}

\author{
Oscar Mañán
}

Resumen. Desde la primigenia publicación de El Capital, vol. iII, se abrió un debate rastreable hasta hoy como «la transformación de valores en precios». Sin pretensiones de exhaustividad se apuntan algunos lados del debate y su posible entendimiento. Las primeras críticas de Bohm-Bawerk ([1896]1974), Dmitriev ([1898]1974) y la síntesis de Bortkiewicz ([1907]1974) resultaron un puntapié inicial. Sraffa ([1960]1965) pretendió terminar la controversia y seguidores como Steedman ([1977]1985) plantearon la «redundancia» de la teoría del valor de Marx para la determinación de las magnitudes de valor, como así de precios y ganancias. La reacción marxista se expresó en Shaikh $(1977,1991)$ al señalar que los problemas de interpretación venían de situar a Marx a continuación de Ricardo sin reconocer una ruptura de éste con los clásicos. Se indaga una «nueva solución» de Mohun (1994) y la defensa del método de Marx encarada por Moseley (1993, 1994).

PALABRAS CLAVE: teoría marxista del valor, transformación de valores a precios, crítica de la teoría marxista.

AвSTRACт. The first publication of Capital (volume ini) started a debate that continues to this day regarding «the transformation problem». While not pretending to be exhaustive, this article points to some possible ways of understanding different sides of this debate. The early critiques made by Bohm-Bawerk ([1896] 1974) and Dmitriev ([1898] 1974) and the synthesis provided by Bortkiewicz ([1907] 1974) constitute the first kick at the can. Sraffa ([1960 1965]) tried to put an end to the controversy; and his followers, like Steedman ([1977] 1985), suggested that Marx's theory of value was «redundant» for determining the magnitude of value, prices and profits. The Marxist reaction was expressed by Shaikh (1977, 1991), who indicated that these problematic interpretations stemmed from placing Marx as a follower of Ricardo, without recognizing the break of the former with the classics. The article explores Mohun's (1994) «new solution», as well as Moseley's $(1993,1994)$ defense of Marx's method.

KEYWORDS: marxist theory of value, transformation of values into prices, criticisms of the Marxist theory.

Oscar Mañán es profesor efectivo del Centro Regional de Profesores del Centro, ANEP-Codicen. Profesor e investigador de la Facultad de Ciencias Económicas y Administración, Universidad de la República, Montevideo, Uruguay. 


\section{TEORÍA MARXISTA Y TRANSFORMACIÓN DE VALORES EN INTRODUCCIÓN}

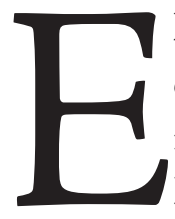

1 debate sobre el «problema» de la transformación de valores a precios cuenta ya con más de cien años de historia y controversias entre los marxistas, siendo la piedra angular que cimentó a los detractores de Marx. La controversia parte con la publicación, a cargo de Engels, del tomo III de El Capital en 1895. Como alguien dijo, se abrió aquí un concurso literario para dar «solución» al mentado problema. $\mathrm{Al}$ año siguiente Eugen von Bohm-Bawerk ([1896]1974) planteó la primera crítica y dos años después V. K. Dmitriev ([1898]1974) presentó la segunda. Tal vez el trabajo más importante - que luego se convertiría en la génesis de la corriente de pensamiento hoy conocida como neoricardianismo- se debe a Ladislaus von Bortkiewicz ([1907]1974); sin prejuicios, todo economista estudioso de la tradición marxista - y que se precie de pertenecer a ella— puede admitir que la «solución» propuesta por ese autor contribuyó a animar la discusión durante el largo siglo xx.

En 1960 aparece un planteamiento cuyo peso «terminó», al decir de sus defensores, con una solución lógica al problema de la transformación, el correspondiente al economista italiano Piero Sraffa ([1960]1965). Empero, hoy varios marxistas piensan que este autor no entendió la esencia del método de Marx - el cual no presenta tales problemas, si se sigue sin pretender falsearlo-; Moseley $(1991,1994)$ es uno de los casos sobresalientes.

Este trabajo no pretende ser exhaustivo. Sólo busca apuntar un debate y varios lados de su posible entendimiento, mismo que lejos de cerrar o enclaustrar una teoría apuntala su lado vital. Se parte de Ian Steedman ([1977]1985), quien sigue la tradición de Sraffa, planteando la «redundancia» de la teoría del valor de Marx para la determinación de las magnitudes de valor, como la de precios y ganancias. Luego se verán las respuestas que la ortodoxia marxista dio a la crítica sraffiana, principalmente desde la óptica de Shaikh (1977, 1991), como también una «nueva solución» propuesta por Simon Mohun (1994). 
Posteriormente, se rescata la defensa del método de Marx que hiciera Moseley (1993, 1994), quien sostiene que si se toman las bases del método de Marx los «errores» aducidos por los neoricardianos se desvanecen. Se concluye con una breve puntualización de las implicaciones que tiene para el análisis marxista del capitalismo tomar los supuestos neoricardianos como base, afirmando la pertinencia del análisis marxista para la realidad capitalista contemporánea.

\section{Las críticas de Steedman}

Steedman comienza por hacer un llamado a los marxistas a realizar un estudio crítico de la transformación de valores en precios, cuestión que los llevará, sin más, a aceptar la solución sraffiana que es «la única lógicamente coherente». Para el autor, la teoría de Sraffa significa una reformulación de Marx, un entierro de sus errores y una reivindicación de aquello que aún es aprovechable en su teoría. Los puntos fundamentales de su crítica, tal y como aparecen en el primer capítulo de su libro, son:

a) Conociendo las condiciones de producción y el salario real pagado a los trabajadores, ambos expresados en cantidades físicas de mercancías, se puede determinar la tasa de ganancia (por supuesto que también los precios de producción).

b) Las cantidades de trabajo materializadas en las ganancias, las que se determinan luego de conocidas las condiciones de producción, no son necesarias para la determinación de la tasa de ganancia.

c) La solución de Marx del llamado problema de la transformación es incorrecta, tanto en lo que afecta a los precios de producción como $-y$ más importante - en lo que atañe a la tasa de ganancia. ${ }^{1}$

\footnotetext{
${ }^{11 /} \mathrm{Al}$ derivarse directamente los precios de producción y la tasa de ganancia sin hacer referencia a la magnitud de valor, el así conocido «problema de la transformación», desaparece.
} 
d) La asignación social de la fuerza de trabajo puede determinarse sin referencia a la magnitud de valor.

e) El vínculo entre plustrabajo y ganancias puede obtenerse en forma también independiente del concepto de valor de Marx.

f) No se vería entonces razón teórica alguna para dilucidar el comportamiento de la tasa de ganancia en el largo plazo.

A pesar de la contundencia que podría representar esta crítica, Steedman piensa que en realidad significa una corrección lógica, que incluso es una herramienta para probar la «afirmación marxista básica: que la explotación capitalista es la fuente de la ganancia» (1985: 35). Partiendo de cantidades físicas de producto y salarios reales, el autor determina los valores y la plusvalía, afirmando que tanto los valores de las mercancías y la fuerza de trabajo dependen de las condiciones técnicas y «sociales» de la producción. Por su parte, sostiene como evidente el carácter de la explotación capitalista, ya que tanto el capital variable (v) como el plusvalor (s) no dependen de las condiciones de las industrias ajenas a la producción de bienes-salario. De aquí deriva el autor una teoría «coherente» de ganancias y precios, pero que es independiente de los valores en el sentido de Marx; entonces $\mathrm{s} / \mathrm{C}+\mathrm{v}$ no será igual a la tasa de ganancia, como tampoco podrán igualarse ganancia total y plusvalía total. Las magnitudes de valor ya no jugarán ningún papel, sino que serán las condiciones físicas de la producción y el salario real las variables que determinen esas magnitudes de valor, luego de haberse elegido la técnica coherente con la maximización de las ganancias, objetivo fundamental del capitalismo. Por lo tanto, «la tasa de ganancia es positiva si y sólo si la plusvalía es positiva». El autor está totalmente consciente del alcance de su proposición al decir: «tal análisis es de importancia decisiva, pero su validez no depende del análisis de la sociedad capitalista basado en magnitudes de valor» (Steedman, 1985: 68).

Sin duda, para no tildarla de ahistórica — cuestión que el autor rechazaría一, se tendría que reconocer su excesivo grado de generalidad. Se volverá sobre el punto para analizar las implicaciones del método neoricardiano. 


\section{Las respuestas de Shaikh}

Shaikh hace un llamado al abordaje del tema de la transformación donde se distinga claramente el lugar de Marx en el pensamiento económico. Al abordar el estudio de Marx como una extensión de los clásicos (fundamentalmente de Ricardo) y adjudicarle las formas metodológicas seguidas por ellos, seguramente se encontrarán grandes discordancias o «redundancias» en su pensamiento. Para el autor, existe una clara ruptura entre Marx y los clásicos, su razonamiento, se basa en una lógica novedosa que significa un avance, algo totalmente nuevo respecto del pensamiento clásico. De aquí que el análisis neoricardiano de las magnitudes de valor deberá entenderse o revisarse a la luz de la conceptualización del valor en Marx.

El método de Marx — siguiendo a Shaikh - busca descubrir la base oculta de la estructura social, siendo la producción el punto de partida «de la práctica social de la sociedad humana», mientras que «la extracción de plusvalía es la base de todas las sociedades de clases», en El Capital particulariza la aplicación de este método al análisis del capitalismo. El capitalismo se distingue de los demás modos de organización de la producción en que el objetivo de ésta es el intercambio. Y se relaciona estrechamente con la producción que además determina sus límites. Por lo tanto, las categorías relativas al intercambio, dinero y precio, así como sus movimientos, están sujetos a las categorías de la producción. En la esfera de la circulación se expresan y resuelven las contradicciones propias de la esfera productiva. La ley del valor es entonces «la manera en que estas contradicciones de la producción dominan el intercambio, tanto cuantitativa como cualitativamente» (Shaikh, 1991: 111).

Desde el punto de partida anterior se divisa una crítica implícita a las cuestiones de conceptualización que evade —o simplemente «oscurece»— el análisis neoricardiano. Shaikh resume en cuatro los tipos de problemas que Steedman le atribuye a la teoría de Marx: a) «el argumento de la redundancia», b) la «inconsistencia», c) la «primacía», y d) la elección de la técnica. 
El primero tiene que ver con el señalamiento de Steedman en cuanto a que la teoría del valor ya no es necesaria para determinar las cantidades de valor, como tampoco ganancias y precios, ya que estas categorías pueden deducirse a partir de las magnitudes de producción física y de los salarios reales. De aquí la nula importancia de los valores trabajo para «determinar» la tasa de ganancia (o precios de producción). Shaikh (1991: 133) sostiene lo contrario: los valores sí determinan los precios y en un «doble sentido» son una forma que adopta el valor, «[...] y magnitudes de estos valores dominan y regulan los movimientos de sus formas precios». A su vez, los precios de producción y ganancias «se basan en la expresión de valores y plusvalía en circulación» $y$ son regulados por las segundas.

Para el autor, el razonamiento neoricardiano adolece de dos debilidades. La primera, la tendencia a ver la producción como un proceso técnico, «solo la "distribución" parece verdaderamente social», y la segunda, «confunden lo real con su apropiación en el pensamiento». Los datos físicos son un «resumen conceptual ex-post de la determinación real» (p. 134), y si se realiza a través de ellos el cálculo conceptual de los valores, sólo se capta su magnitud real.

El argumento de inconsistencia de Steedman — dice Shaikh — tiene dos puntas: una, la que se acaba de describir, y otra, la que tiene que ver con las desviaciones precio-valor. Quizá el argumento más débil, en la respuesta de Shaikh, ${ }^{2}$ apunta a que la suma de valor no se altera en el intercambio; sin embargo, es aquí donde se producen las transferencias de valor que originan las desviaciones precio-valor que están en la base de la discrepancia entre suma de ganancias y suma de plusvalía, «sin violar la ley de la conservación del valor en el intercambio» (p. 137). Critica la ausencia en el razonamiento neoricardiano del concepto de autonomía relativa de la circulación, en cuya esfera puede efectivamente observarse la discrepancia entre la tasa de ganancia en valor $y$

${ }^{\text {/2/ }} \mathrm{El}$ adjetivo débil se justifica porque ingresa nuevos conceptos como «precios directos»y «ganancias directas» que sólo complican su análisis, porque el mismo se despende en forma lineal del planteamiento de Marx. 
aquélla transformada que calculara Sraffa. Empero, hay que hacer hincapié en que la desviación no es para nada arbitraria, ya que ésta se mueve con la cadencia y los límites que el movimiento de la tasa de plusvalía le imprime. Es aquí que la deficiencia conceptual — para Shaikh — emerge de forma definitoria por encima de los mantos algebraicos que buscan emparentar los argumentos con la lógica, quedando al desnudo las insuficiencias de su método.

En lo que hace al argumento de la primacía, donde Steedman sostiene que la tasa de ganancia en valor es desconocida para los capitalistas, ergo, no es importante para el análisis del capitalismo. Al decir de Shaikh, «de un plumazo Steedman tira por la borda toda la ciencia» en el sentido de que hay una exacta correspondencia entre esencia y apariencia. Shaikh argumenta que por más que no existan como tales en la circulación, precios de producción y tasa media de ganancia son importantes ya que regulan las fluctuaciones de los precios de mercado y las tasas de ganancia. Por ello, los valores y las tasas de ganancias en valor son más que importantes porque «dominan y regulan a su vez los precios de producción y la tasa uniforme de ganancia» (p. 141). El impedimento de Steedman, para no ver lo anterior, estaría en el concepto neoclásico de equilibrio, que el autor remplaza por el concepto de regulación tendencial de los valores, tirando por tierra la contradicción básica del capitalismo. Es así que el proceso de abstracción se convierte en una «idealización» al mejor estilo de «la economía vulgar».

El llamado argumento de la elección de la técnica hace referencia a que los capitalistas siempre eligen la técnica coherente con la mayor tasa de ganancia a precios y salarios dados. Se desprende, entonces, que una elección de técnica se hace, sí y sólo sí, implica una mayor tasa de ganancia, cuestión que ajusta al alza la tasa media de ganancia. Por lo tanto, las magnitudes de valor estarán determinadas por la elección de la técnica y la tasa de ganancia se determinará anteriormente a dichas magnitudes.

$¿$ En qué consisten las confusiones neoricardianas? Primeramente, que «nunca los precios y las tasas de ganancia de mercado son iguales a los precios de producción y a la tasa uniforme de ganancia», lo que lleva a pensar 
en la dificultad de poder elegir la combinación que rinda una tasa media de ganancia mayor. Otro error de Steedman estaría en no tomar en cuenta que la evaluación de los métodos de producción se realiza basada en «ganancias potenciales», no sólo evaluadas por los precios anticipados, sino también «por el funcionamiento anticipado del proceso de trabajo y las rentas esperadas». Es entonces que las ganancias capitalistas responden a la «potencial creación de valor y plusvalía en la producción», y a su «realización estimada en la circulación», y no sólo a una estimación de costos y ganancias en la circulación. Por último, se desprende que el concepto de ganancia de Steedman —insinúa Shaikh-, refiere más al concepto neoclásico, es decir, que ésta forma parte de los costos de producción, no resultando claro que ajuste a la idea de excedente sobre costos que está en la base del razonamiento marxista. Por lo tanto, el argumento neoricardiano que plantea la espuriedad en la relación de la tasa de ganancia y la composición orgánica queda a su vez «falseado». ${ }^{3}$

\section{El planteamiento de Mohun}

Mohun, por su parte, también argumenta, en la línea anterior, que el pensamiento neoricardiano interpreta a Marx a través de los postulados a priori de Ricardo. Entonces, las formas del dinero pasan desapercibidas al mismo tiempo que la mercancía dinero es desplazada de la circulación. El dinero aparece como una magnitud de cuenta del trabajo incorporado en el valor e «irrelevante para la explicación teórica» (p. 405). Es así que el «sistema de valores» viene a determinar «las razones de trabajo incorporado»y el «sistema

${ }^{13 /}$ Las páginas entre paréntesis y las expresiones entrecomilladas pertenecen al capítulo tres de $\mathrm{Va}$ lor, Acumulación y Crisis que se intitula «Notas críticas sobre algunos argumentos Neoricardianos y Neoclásicos» (pp. 131-148). También puede encontrarse críticas explícitas a Steedman en el capítulo seis, «Crisis económicas y tasa decreciente de ganancia» y en las sesiones «Economía Política y Capitalismo: Notas sobre la Teoría de la Crisis de Dobb» (pp. 299-325) y «Debate sobre la elección de la técnica» (pp. 328-341). 
de precios, además de las tasas de intercambio en el mercado». Para Mohun, este pasaje de valores a sumas de dinero requiere un análisis más concreto y a un nivel de abstracción menor que aquél que se utiliza para determinar estos valores propiamente. Dado que la competencia capitalista «modifica» estos valores - que fueron apropiados para el análisis del capital en general一, ahora se convierten en precios de producción, y a pesar de que siguen siendo valores, les corresponde una expresión en horas de trabajo socialmente necesario.

En la tradición ricardianana - dice Mohun-, el valor del dinero es el factor de conversión del trabajo abstracto en «valores monetarios»; sin embargo, y a pesar de lo anterior, éste se supone constante, pasando entonces del problema de la transformación al problema del pasaje de los valores monetarios a los precios de producción. Más aún, si encima se reduce el valor de la fuerza de trabajo al valor de una canasta de consumo de subsistencia, tanto con intercambio desigual o igualitario, aparece una identificación del valor definido en términos de trabajo con una particular distribución de los tiempos de trabajo mediante un sistema de precios. Este razonamiento supone un intercambio en condiciones igualitarias entre el valor de la fuerza de trabajo - expresada en valor monetario - y una canasta de consumo que satisfaga la demanda de subsistencia. De aquí la «confusión» entre el trabajo expresado en valor con una distribución particular de dicho valor realizado por el sistema de precios, que lleva a «eliminar la distinción entre capital variable como capital y el salario en las manos del trabajador como una suma de ingresos».

Mohun plantea una solución propia que llama «teoría del trabajo abstracto», la que superaría la propuesta ricardiana, poniendo a la forma del valor en el centro del análisis en vez de separar los distintos niveles «epistemológicos de análisis». Aquí el autor distingue al valor como: $a$ ) el tiempo de trabajo en su apariencia de dinero y $b$ ) valor monetario correspondiente al valor de la mercancía dinero. El valor del dinero está dado por «la relación entre el tiempo de trabajo social total gastado y el valor monetario del producto creado en dicho tiempo de trabajo». Dado que el sistema de precios no reparte el tiempo de trabajo con proporcionalidad exacta al tiempo de trabajo requerido para la 
producción de una mercancía, habrá una discrepancia entre el valor del dinero - donde se expresa el valor de las distintas mercancías- y el tiempo de trabajo empleado para la producción de dicha mercancía-dinero. El dinero no es inmediatamente la expresión del tiempo social de trabajo requerido para la producción de una mercancía, es sí una representación de dicho tiempo de trabajo, pero además una forma de valor.

En la tradición de Von Bortkiewicz — prosigue Mohun - no existe elección de consumo, ya que los trabajadores reciben una canasta de subsistencia dada (salario real dado). Sin embargo, lo que ocurre es que los trabajadores son remunerados con una suma de dinero (no una canasta de subsistencia). He aquí «el objetivo del conflicto de clase», donde capitalistas y trabajadores miden fuerza: uno por elevar su ganancia y el otro por mantener un valor tal de la fuerza de trabajo acorde con sus necesidades (construidas socialmente, aunque se olvida de la dinámica). No habría razón alguna para suponer que todos los obreros consumen la misma canasta de subsistencia, o que consumen todo su salario, aunque éste último debe cumplir la condición de reproducción de la fuerza de trabajo como tal. Para el autor, el error neoricardiano viene del tratamiento de Marx en El Capital, tomo uno, donde el autor «se basa en el proceso de producción individual» en aras de explicar la creación de plusvalía y dilucidar qué parte de ésta se convertirá en capital (el tema será retomado con la crítica de Moseley).

Aquí se supone intercambios proporcionales a los valores - tiempo de trabajo incorporado en la producción-, mientras que los seguidores de Bortkiewicz plantean que dicho trabajo gastado debe transformarse en precios de producción, mostrando que las cantidades invariables no se desprenden de este proceso, lo que les lleva al rechazo de la ley del valor. Mohun, por su parte, afirma que, con su «nuevo enfoque», al tratar el producto neto agregado como el total de valor producido por el trabajo productivo, también trata su forma dinero de una forma más general - y de manera central para el análisis del capitalismo.

En el nuevo planteamiento, los precios son el medio por el cual se distribuyen los valores agregados como una forma de valor, no dependiendo así de 
ningún supuesto acerca del intercambio. Su punto de partida, «una apropiada definición de los agregados» (que no varían) a partir de la «definición de la teoría del valor trabajo», definiendo estos agregados para «cualquiera sea la distribución de valor del sistema de precios». No habrá aquí «transformación alguna de valores (monetarios) a precios».

Con la propuesta anterior — concluye el autor-, la teoría del valor trabajo deja de ser «metafísica» aportando una serie de significados a la relación entre valores y precios. Expresado en tres ecuaciones básicas - (2), (3) y (4)—, muestra que existe un producto debido a los trabajadores, que es posible la determinación de las horas de trabajo agregadas, y que al definir el valor del dinero se tiene que el valor de la fuerza de trabajo está dado por el salario multiplicado por dicho valor del dinero. Aquí las ganancias son plustrabajo no pagado y la tasa de explotación está «bien definida» y con independencia de la producción conjunta o la elección de la técnica. Se llega así — dice Mohun - a una teoría del valor trabajo «estructurada por los agregados macroeconómicos invariables de valor agregado y su división, en salarios y ganancias», que será más «operacional y consistente» que aquélla que se desprende de la tradición de Bortkiewicz.

\section{La Revisión de Moseley}

Moseley $(1993: 157)$ pasa recuento al enfoque neoricardiano y su principal objetivo es demostrar que el debate del «problema de la transformación no ha prestado la suficiente atención al método lógico empleado por Marx en los tres volúmenes de El Capital». En especial, la interpretación neoricardiana, según Moseley, «[...] atribuye a la teoría de Marx un método lógico [...] que es en esencia el método de la teoría de la producción lineal» (p. 157).

$\mathrm{El}$ autor argumenta que debido a que ambos métodos son diferentes es obvio que también los resultados lo serán y esto explica tan larga controversia. Las implicaciones serán, entonces, las ya vistas: a) que sólo una de las igualdades agregadas proclamadas por Marx — precios agregados = valores agregados $\mathrm{y}$ 
ganancias agregadas = agregados de plusvalía - se comprueba al mismo tiempo; b) la tasa de ganancia en precios no coincide con la tasa de ganancia en valor derivada del sistema de precios — además presenta tendencias divergentes-, y c) el argumento de la «redundancia» ya discutido — donde los mismos precios y la tasa de ganancia pueden obtenerse a partir de las cantidades físicas-.

Ambos enfoques, neoricardiano y marxista, son disímiles. Básicamente se trata del «orden de la determinación entre magnitudes económicas agregadas (como total de precios y total de ganancias) y la correspondientes magnitudes individuales» (p. 159). Para la interpretación neoricardiana se determinan las variables individuales y luego la suma que forma - a posteriori- el agregado. Sin embargo, el método de Marx determina las variables agregadas (capital social) primero y con independencia de las magnitudes individuales.

Esta interpretación, opuesta al método de Marx, se basa en un también largo debate sobre los niveles de abstracción en que se piensa y escribe el tomo uno de El Capital y aquéllos correspondientes al tomo tres. ${ }^{4}$ En el primer libro de El Capital, Marx define las variables a nivel agregado, ${ }^{5}$ para lo cuál no necesita

14/ Vale reiterar aclaraciones de Marx sobre la discordancia entre el método de investigación y el correspondiente al de exposición. Siguiendo a Marx y como aparece en «El método de la economía política», el comienzo de todo proceso de investigación es la realidad objetiva, tal y como aparece descripta en el tomo tres (el análisis de la competencia, el movimiento del capital social, pero con sus particularidades en acción). Empero, es menester realizar un proceso de abstracción para poder reproducir la complejidad real en el pensamiento, para así conocer sus múltiples determinaciones y poder encontrar la esencia oculta de los fenómenos (las leyes fundamentales que explican el movimiento de dicha complejidad). Ese proceso de abstracción y simplificación, que llevan a conocer las propiedades fundamentales del capital social (aunque bajo la simplicidad de la estática) para apuntar qué es lo que determina su lógica de crecimiento y cuáles son las condiciones que la promueven (esto se encuentra en el tomo primero).

15/ Véase la nota a pie de la página número tres, en el apéndice de Moseley (1993: 182). Existe incluso una tendencia de los marxistas a interpretar el planteamiento de Marx en el tomo uno como un análisis del capital individual, tal como si fuera el método de la teoría del valor neoclásica, la cuál demuestra su fuerza explicativa del comportamiento del «agente (consumidor) representativo»; cargando luego con los consabidos problemas de «agregación» (aplican aquí las críticas de Garegnani, Sraffa, entre muchos otros). 
ningún supuesto (a la Sweezy) ni de composición orgánica igual de los capitales, ni que los precios de las mercancías individuales sean iguales. Lo anterior, debido a que las variables agregadas (precios y ganancias) se determinan «antes y con independencia» de las magnitudes individuales. En el libro tercero, Marx no levanta supuestos sino que cambia el nivel de abstracción, pasa del análisis del capital en general a la consideración de los muchos capitales, para con ello explicar la distribución de la plusvalía creada por el movimiento del capital social. Aquí las magnitudes agregadas son tomadas como dadas debido a que ya se explicó (en el volúmen primero) su determinación. Al decir de Marx:

[...] la ganancia de los capitalistas como clase, o la ganancia del capital como un todo, debe de existir antes de que pueda ser distribuido, y es extremadamente absurdo tratar de explicar sus orígenes por su distribución (Marx, 1973, citado por Moseley, p. 161).

Otra gran diferencia de la interpretación ricardiana del método de Marx - según Moseley - está dada por los supuestos o puntos de partida que se toman como dados. Para la corriente neoricardiana dichos supuestos son: a) cantidades físicas y b) salarios reales, ya vistos. Sin embargo, los puntos de partida de Marx serán: a) cantidades de dinero y $b$ ) cantidades de trabajo abstracto (o cantidades de dinero que representan horas de trabajo abstracto). Marx — rescata Moseley - comienza por la suma de dinero como resultado del proceso de circulación de capital y este dinero se transformará en capital — capital definido como dinero que hace más dinero-. En cambio, el análisis neoricardiano parte de un monto de insumos que se transforma en capital, para posteriormente comenzar el proceso de «the production of commodities by means of comodities» (producción de mercancías mediante mercancías). Lo cual es muy diferente.

Con dichas consideraciones de método, el autor sostiene que las dos igualdades agregadas de Marx son ciertas simultáneamente y que la «determinación de los precios individuales no alteran la tasa de ganancia», en virtud 
de que dicha tasa de ganancia se toma como dada en la determinación de los mencionados precios individuales. La crítica de la «redundancia» no aplica debido a que «el valor de las mercancías no puede derivarse de las condiciones técnicas de la producción», por lo menos bajo el método de Marx. Para este pensador - subraya Moseley - el concepto de trabajo abstracto no es comparable a «los requerimientos de trabajo corriente incluidos en las condiciones técnicas de producción» de la tradición neoricardiana, ya que este último no distingue entre tipos de trabajo e intensidad de éstos. Por otra parte, «el trabajo pasado» que constituye el capital constante no podría igualarse al trabajo gastado en los medios de producción. Para Marx, entonces, «el valor de las mercancías» no resulta «an unnecessary detour» (un desvío innecesario) de las condiciones técnicas de producción a los precios de las mercancías, sino un presupuesto «indispensable» de su «teoría de la ganancia».

Para concluir, Moseley encara una crítica a las «nuevas soluciones» (Foley, 1982; Duménil, 1980, 1983; Lipietz, 1982; podría incluirse a Mohun, 1994), las cuales tienen una «inconsistencia metodológica», ésta radica en un tratamiento diferencial para el capital constante y el capital variable. El primero se deriva de las cantidades físicas, mientras que el segundo se toma como dado en términos monetarios. Según Moseley, tanto el capital constante como el variable son parte del capital invertido por los capitalistas y como tal deberían tener un tratamiento uniforme en cuanto a las unidades de medida. De lo contrario, dicha argumentación tendría que refutar los presupuestos de Marx en el tratamiento del capital que comienza con una suma de dinero $\left(\mathrm{M}-\mathrm{C}-\mathrm{M}^{\prime}\right)$, o bien replantear una argumentación para el esquema lógico que el autor sigue en su exposición de los diferentes libros de El Capital. Moseley señala a su vez que dicha corriente representa una «opción intermedia» entre el planteamiento marxista (variables en términos de dinero) y el planteamiento neoricardiano (en términos de cantidades físicas). Shaikh, por su parte, es más drástico al evaluar en particular la propuesta de Mohun. Para dicho autor esta solución sucumbe de antemano a las críticas neoricardianas. 


\section{Algunas implicaciones del}

\section{ENFOQUE NEORICARDIANO}

Sin duda, nadie en su sano juicio puede ignorar el impacto que tuvo el planteamiento de Sraffa - equivocado o no- en el debate interno de la teoría económica - marginalista y marxista-, habiendo representado una fuente de reflexión y de contraposición de ideas, cuestión que trae a la vida a cualquier disciplina científica.

Las implicaciones son muchas, tanto en lo que hace a la teoría económica propiamente, como aquéllas que, derivadas de la teoría económica, impactan a otros ámbitos de la práctica social, por ejemplo, la política. En 1978 se reunieron en la Universidad de Módena varios académicos para enterrar la teoría perimida ante el «revolucionario» planteamiento del economista italiano. El gran debate aludió a cuál es la parte de la teoría marxista que sobrevive a la confrontación con la nueva teoría de la producción conjunta. Vayan algunas de éstas implicaciones teóricas que seguramente están en la base del «reformismo político».

Si se rechaza el concepto de valor, debería abandonarse también el de plusvalía. Por lo tanto, el concepto de explotación - por ser una relación en términos de valor - tan arraigado en la teoría marxista, quedaría sin fundamento (Guillén Romo, 1988). Sin embargo, muchos ricardianos se obstinan en mantener la teoría de la explotación, más allá de que sería muy difícil la deducción lógica de ésta en el contexto del método de la producción conjunta. Garegnani es uno de ellos, quien afirma que «la proposición que se refiere a la existencia de la explotación del trabajo en una sociedad capitalista no depende de ninguna manera de la validez de toda la proposición teórica fundada en la noción de excedente» (Garegnani, 1979: 57).

Steedman (1985: 68) muestra claramente esta visión de excedente a que se refieren los seguidores de Ricardo. Con el método de análisis seguido por estos autores, el excedente aparece como una cantidad de producto no consumido, dada la canasta de subsistencia (salario real). Es decir, en un sistema 
económico donde se produce más de lo que se consume, y existe algún impedimento para que los productores directos se apropien del producto, habrá entonces explotación. Marx respondería:

El trabajo excedente no fue inventado por el capital. Dondequiera que una parte de la sociedad posea el monopolio de los medios de producción nos encontramos con el fenómeno de que el trabajador, libre o esclavizado, tiene que añadir al tiempo de trabajo necesario para poder vivir una cantidad de tiempo suplementario, durante el cual trabaja para producir los medios de vida destinados al propietario de los medios de producción, dando lo mismo que este propietario sea el propietario aristócrata ateniense, el teócrata etrusco, el civis romanus, el barón normando, el esclavista norteamericano, el bayardo de la Valaquia, el terrateniente moderno, o el capitalista (Marx; [1867] 1972: 180-181).

Seguramente es fácil acordar que esta visión ricardiana de excedente es muy diferente de la que podría emparentarse con el concepto de explotación propio del sistema capitalista. El excedente existe cuando el plustrabajo se convierte en plusvalía, lo que sucede al encontrarse el propietario de los medios de producción frente al obrero libre, ambos entran en una relación de explotación con el objeto de producir mercancías, y ello ocurre cuando los medios de producción toman la forma de capital. La idea neoricardiana de excedente, como fue dicho (cantidad de producto no consumido), no implica el concepto de explotación ya que los salarios reales (canasta de consumo de subsistencia) están dados, y será entonces la elección de la técnica hecha por el capitalista (coherente con una tasa de ganancia mayor) la que determine dicho excedente.

Los autores de esta corriente no llegan a quitar el velo de las relaciones en términos de magnitudes de productos físicos para poder ver el fondo, es decir, las relaciones sociales de producción.

El economista italiano Napoleoni (1979 a y b) festejó eufórico el cierre del sistema de Marx presentado por Sraffa —entre otros, i.e., Dobb (1970, 
1975)-. A pesar de acordar sobre las consecuencias del enfoque neoricardiano en la teoría marxista del valor, sostiene, curiosamente, que ésta debe seguir discutiéndose a nivel «filosófico». Lucio Colleti (1979) acepta algunos cuestionamientos a la teoría marxista del valor, empero, y esgrimiendo más coherencia que el anterior, manifiesta que «sin el concepto de valor no se puede definir en Marx ni siquiera los conceptos de dinero y de capital, mucho menos los conceptos de mercado y de competencia».

Válida resulta la siguiente crítica en cuanto a que:

[...] todos los intentos que han hecho los neoricardianos por superar o corregir al marxismo sólo han conducido a deformaciones superficiales; a la trivialidad y al eclecticismo más burdo al estilo Garegnani, cuando proclama que la explicación de la ganancia y los precios pueden obtenerse a partir de Sraffa, mientras que para explicar la acumulación y la crisis se debería recurrir a El Capital de Marx (Guillén, 1988: 237).

Si bien, como fue planteado antes, los ciento diecisiete años de controversia han sido un motor de la discusión sobre la fuerza y los límites de la teoría, hace ya tiempo que no hay avances en el sentido del método de Marx. Desde el lado de los seguidores de Marx, quizá los trabajos más contundentes y reveladores sobre la reivindicación de su método tienen ya más de veinte años. Nos referimos a los trabajos de Rosdolsky (1978) y la conocida solución de Salama y Valier (1973) y Salama (1975). El planteamiento de Anwar Shaikh adolece de claridad y no va más allá de lo señalado por la ortodoxia marxista, y más aún, cuando plantea el método de iteración da la impresión que de alguna manera acepta el error de Bortkiewicz. Por su parte, Moseley logra mostrar en forma por demás sugerente una puntualización de las diferencias entre el método de Sraffa y el de Marx, poniendo el énfasis en el tratamiento de las variables —al estilo Rosdolsky y Salama—; cuestión que también aplica para cuestionar duramente las «nuevas soluciones», como la de Mohun aquí comentada. 
Para concluir el punto, valga reafirmar que las críticas destructivas o correctivas a la teoría del valor resultan, más que nada, un deseo de apuntar su obsolescencia. Sin embargo, si dichas hipótesis parten de una comprensión falsa del problema, ergo, sus conclusiones son, en el mejor de los casos, dudosas. Debido a que las mercancías se expresan «inmediatamente en dinero», incluso antes de que éste las haga circular, el valor de cambio no será entonces equivalente al valor ni a su magnitud, «sólo es una forma fenoménica del mismo». El precio de mercado, forma en que aparece el valor, «fluctúa alrededor del precio de producción de mercado». Con dicho razonamiento $-y$ siguiendo a Salama-, el costo de producción se expresa «inmediatamente en términos monetarios» $y$ así los precios de producción serán «la expresión del valor en la esfera de los capitales numerosos» (Rosdolsky, 1978). Por lo tanto, el supuesto «error» de Marx no existe.

El llamado «problema de la transformación» es entonces un cambio en el nivel de abstracción, un pasaje del análisis del capital social (en general) a la esfera de los capitales numerosos, es decir, a la esfera de la competencia capitalista. Es esta última la esfera de las apariencias; sin embargo, al realizar el proceso de abstracción que permite dilucidar el movimiento del capital social y sus determinantes, se puede ahora construir el «concreto pensado» que da cuenta de una unidad conceptual, de la diversidad de lo real. Los precios de producción como tales son una expresión de la ley del valor, por lo tanto, es posible a través de ellos entender la anatomía de la sociedad capitalista, descubrir sus contradicciones y tener una guía para la acción politica.

La explotación no es un fenómeno individual sino que es un hecho de clase. Marina Bianchi plantea que para el cálculo de valores «el antes» $\mathrm{y}$ «el después» de Marx «tiene un significado social preciso»: explicar la génesis de la tasa media de ganancia y los precios de producción; «romper con ese específico nexo significa [...] no comprender el problema peculiar de Marx y colocarse, en cambio, directamente a continuación de Ricardo» (Bianchi, 1975: 36-37).

Dada las consideraciones anteriores, para los neoricardianos el antagonismo de clase en el capitalismo se reduce a una relación distributiva. En- 
tonces, las soluciones son fáciles y consisten en mejorar las condiciones de la distribución. Además de no tener herramientas teóricas para poner al descubierto distintas formas de antagonismos, como son los que se dan entre las clases sociales, la lucha por el excedente en la esfera de la circulación pasa a ser lo «verdaderamente social» de esta teoría, convirtiéndose así en la base teórica más fuerte del pensamiento reformista burgués.

\section{LA TEORÍA DE MARX, ¿VIVA O MUERTA?}

La teoría de Marx - que quizá habría que distinguirla de la teoría marxista- representa un método de análisis social muy poderoso. Si bien, cuando se recorta dicha teoría social en diversos subconjuntos — al estilo de, i.e., Blaug (1980) - no siempre el subproducto, como pudo verse en el caso concreto del planteamiento neoricardiano, resulta coherente con sus objetivos originales. Lo anterior es un gran problema con la teoría marxista, ya que una infinidad de seguidores de Marx han tratado de refuncionalizar algunos conceptos clave de la teoría en otros marcos teóricos diferentes, cuestión que - en el mejor de los casos - termina por quitarle el sentido y la fuerza que dichos conceptos mostraban en su contexto teórico original. Muchas veces el resultado es aún peor, generando serias contradicciones dentro del nuevo marco teórico-conceptual. Con estas salvedades, cuando se refiere a la teoría de Marx, debería significarse su obra en general, la cual, como se intentó mostrar, a pesar de los innumerables ataques, mantiene una coherencia lógica y una fuerza explicativa poco frecuente en el conjunto de la teoría social.

Sin embargo, y como es normal en cualquier teoría que goce de buena salud, existen muchos planteamientos que necesitan desarrollarse y articularse dentro del contexto de la teoría a nivel más abstracto. Quizá la idea kuhniana de paradigma da mejor cuenta de la teoría de Marx que las fronteras propias de las ciencias sociales (sociología, economía, etcétera). En cuanto tal, esta teoría genera todos los días problemas nuevos y soluciones diferentes 
para aquellos investigadores que, en base a su herramental, afrontan el estudio de la realidad contemporánea. Por ejemplo, y dada la preocupación concreta de la teoría económica marxista, ésta no ha desarrollado un cuerpo teórico competitivo (con las demás teorías) en ámbitos como la macroeconomía, la teoría del crecimiento, la teoría del desarrollo, entre otros. Si bien es cierto que ha «prestado» —en el sentido ya señalado-gran parte de su conceptualización, y que la misma, usada —indiscriminadamente- es fundamental para el desarrollo de las anteriores subdivisiones de la teoría económica, también lo es que no existe una teoría macro marxista, una teoría del crecimiento y demás, con suficiente sistematización que permita al estudioso exigido por la actual división del trabajo acceder de forma más expedita —en vez de rastrear en la obra de Marx o, en su defecto, en los manuales- las soluciones a los problemas más comunes que le plantea su disciplina.

Por supuesto, el desafío es importante, pero sin duda el método marxista es una herramienta capaz de articular dichas necesidades con su marco general más abstracto. Las ventajas que tendría un esfuerzo de este tipo saltan a la vista, máxime cuando se mira rápidamente a la teoría económica dominante.

La teoría del equilibrio general y sus desarrollos actuales - fundamentalmente aquellos que engloban los aportes de Hicks y Samuelson- parecerían ser «la teoría» más robusta a los ojos de la moda de hoy. Sin embargo, al contraponer la tradición neoclásica hoy dominante con su homóloga clásica, puede observarse que la primera presenta problemas insolubles y descansa en supuestos tales que, de antemano, invalidarían cualquier resultado. La crítica de Sraffa a la teoría neoclásica y marginalista es contundente, no es serio suponer que el funcionamiento de toda la economía tiene el mismo comportamiento que un agente individual. Los problemas de agregación y los supuestos que «hacen posible» dicha agregación tornan dudosa cualquier conclusión.

La teoría keynesiana, por su parte, tiene un desarrollo teórico insuficiente, presenta un interesante avance en la perspectiva macroeconómica, aunque a su vez evidencia una gran dependencia de los supuestos neoclásicos fuertes. Su propuesta metodológica, coincidente con el marco neoclásico, resulta un 
escollo insalvable para compatibilizar la crítica macroeconómica en el marco de los microfundamentos que comparte con la tradición neoclásica.

Por último, es necesario reafirmar que la teoría marxista tiene aún mucho que decir. Primero, sincerarse respecto a sus debilidades: de la teoría de la competencia capitalista «se sabe poco», de las decisiones de los capitales individuales y su confrontación se conocen abstracciones muy generales que quizá no permiten conclusiones de carácter contundente. El tema de la planificación del desarrollo, que a priori presenta ventajas comparativas en el papel respecto al sistema de mercado, tuvo siempre dificultades concretas que terminaron imponiéndose a la dinámica real. El logro de la eficiencia social en un sistema de planificación centralizada sigue siendo un «misterio» a develar. Estos, como otros tópicos, que seguramente saldrán de los investigadores que se asomen al estudio de la teoría y método marxista y su aplicación al análisis de las sociedades concretas, serán la base del porvenir de la teoría marxista.

\section{Referencias}

Bianchi, Marina (1975), La teoría del valor desde los clásicos a Marx, Madrid, Alberto Corazón Editor.

Blaug, Mark (1985), Teoría económica en retrospectiva, México, Fondo de Cultura Económica.

Dовв, Maurice (1975), Teoría del valor y de la distribución desde Adam Smith, México, Siglo XxI.

(1970), «The Sraffa system and the critique of the neoclassical theory of distribution», De Economist, vol. 118, núm 4.

Böнm-BAwerk, Eugen Von ([1896]1974), «La conclusión del sistema de Marx», en Paul Sweezy (editor), Economía burguesa y economía marxista, Cuadernos de Pasado y Presente, núm. 49, Buenos Aires, Siglo XxI.

BortKiewicz, Ladislaus ([1907]1974), «Contribución a una rectificación de los fundamentos de la construcción teórica de Marx en el volumen iII 
de El Capital», en Paul Sweezy (editor), Economía burguesa y economía marxista, Cuadernos de Pasado y Presente, núm. 49, Buenos Aires, Siglo XXI.

Colettri, Lucio (1979), «Valor y dialéctica en Marx», en Pierangelo Garegnani (compilador), Debate sobre la teoría marxista del valor, Cuadernos de Pasado y Presente, núm. 82, México, Siglo xxI.

Dimitriev, Vladimir Karpovitch ([1898]1974), Economic Essays on Value, Competition and Utility, Cambridge, Cambridge University Press.

Duménil, Gérard (1980), De la valeur aux prix de production, Paris, Economica.

Foley, Duncan (1982), «The Value of Money, the Value of Labor-Power, and the Marxian

Transformation Problem», Review of Radical Political Economics, núm.14.

Garegnani, Pierangelo (1979), «La realidad de la explotación I, II, in I», Debate sobre la teoría marxista del valor. Cuadernos de Pasado y Presente, núm. 82, México, Siglo xxI.

Guillén Romo, Héctor (1988), Lecciones de economía marxista, México, Secretaría de Educación Pública, Fondo de Cultura Económica.

Lipietz, Alain (1982), «The "So-Called Transformation Problem” Revisited», Journal of Economic Theory, núm. 26.

Marx, Karl ([1867]1972), El Capital, México, Fondo de Cultura Económica. Mohun, Simon (1994), «A re(in)statement of the labour theory of value», Cambridge Journal of Economics, núm. 18.

Moseley, Fred (1993), «Marx's Logical Method and the "Transformation Problem"», en Fred Moseley (editor), Marx's Method in Capital: a reexamination, Atlantic Highlands, NJ, Humanities Press International, Inc. (1994), «Capital in General and Marx’s Logical Method: a Response to Heinrich's Critique», Capital E Class, núm. 56.

Napolioni, Claudio (1979), «El enigma del valor», en Pieragelo Garegnani (compilador), Debate sobre la teoría marxista del valor. Cuadernos de Pasado y Presente, núm. 82, México, Siglo xxi. 
Napolioni, Claudio (1979), «El Marx inútil de Lippi», en Pierangelo Garegnani (compilador), Debate sobre la teoría marxista del valor. Cuadernos de Pasado y Presente, núm. 82, México, Siglo xxi.

Rosdolsky, Roman (1978), Génesis y Estructura de El Capital de Marx, México, Siglo xxI.

Salama, Pierre y Jacques Valier (1973), Una introducción a la economía politica, México, Era.

Salama, Pierre (1975), Sobre el Valor, México, Era.

SHAIKH, Anwar (1977), «Marx's Theory of Value and the "Transformation Problem"», en J. Schwartz (editor), The Subtle Anatomy of Capitalism, Santa Mónica, Goodyear.

(1991), Valor, acumulación y crisis, Bogotá, Tercer Mundo.

Sraffa, Piero ([1960]1965), Production of Commodities by Means of Commodities, Cambridge, Cambridge University Press (traducción de 1965, Producción de mercancías por medio de mercancías, Barcelona, Oikos).

Steedman, Ian ([1977]1985), Marx, Sraffa y el problema de la transformación, México, Fondo de Cultura Económica (Marx after Sraffa, London, New Left Books). 